\title{
Effect of the spinal apelin-APJ system on the pathogenesis of chronic constriction injury-induced neuropathic pain in rats
}

\author{
QINGMING XIONG ${ }^{1-3^{*}}$, WANYOU HE ${ }^{1}$, HANBING WANG $^{1 *}$, JUN ZHOU ${ }^{1}$, \\ YAJUN ZHANG ${ }^{1}$, JIAN HE ${ }^{1}$, CHENGXIANG YANG ${ }^{1}$ and BIN ZHANG ${ }^{1}$ \\ ${ }^{1}$ Department of Anesthesiology, The First People's Hospital of Foshan, Foshan, Guangdong 528000; \\ ${ }^{2}$ Jiangsu Key Laboratory of Anesthesiology; ${ }^{3}$ Jiangsu Key Laboratory of Anesthesia and Analgesia \\ Application Technology, Xuzhou Medical College, Xuzhou, Jiangsu 221000, P.R. China
}

Received March 31, 2016; Accepted March 8, 2017

DOI: $10.3892 / \mathrm{mmr} .2017 .6734$

\begin{abstract}
Apelin is hypothesized to serve a dual function in pain processing. Spinal administration of apelin induces hyperalgesia, while opioid receptors are implicated in the antinociceptive effects of apelin in acute nociceptive models. However, whether the apelin-apelin receptor (APJ) system is involved in neuropathic pain remains to be elucidated. The present study aimed to evaluate the impact and mechanism of the spinal apelin-APJ system in neuropathic pain. Chronic constriction injury (CCI) of the sciatic nerve produced sustained spinal apelin and APJ upregulation, which was associated with mechanical allodynia and heat hyperalgesia development in the hind-paw plantar surface. Immunofluorescence demonstrated that apelin and APJ were localized to the superficial dorsal horns. In order to further clarify the function of the apelin-APJ system, a single intrathecal administration of ML221, an APJ antagonist, was used; this transiently reduced CCI-induced pain hypersensitivity. However, apelin-13 (the isoform which binds most strongly to APJ) exhibited no effect on the nociceptive response, suggesting an essential role for the spinal apelin-APJ system in neuropathic pain sensitization. The present study demonstrated that a single application of ML221 alleviated mechanical allodynia and heat hyperalgesia 7 days following CCI, in a dose-dependent manner. Intraspinal delivery of ML221, at the onset of and in fully-established neuropathic pain, persistently attenuated CCI-induced pain hypersensitivity, indicating that the apelin-APJ system was involved in initiating and maintaining pain. It was demonstrated, using immunoblotting, that intrathecal ML221
\end{abstract}

Correspondence to: Dr Bin Zhang, Department of Anesthesiology, The First People's Hospital of Foshan, 81 North Lingnan Road, Foshan, Guangdong 528000, P.R. China

E-mail: 1124873200@qq.com

*Contributed equally

Key words: apelin, apelin receptor, neuropathic pain, chronic constriction injury, spinal cord, dorsal horn downregulated phosphorylated extracellular signal-related kinase (ERK) in the rat spinal cord dorsal horn, suggesting that the effect of apelin on neuropathic pain may be mediated via ERK signaling. The results of the present study suggested that the spinal apelin-APJ system may drive neuropathic pain. Inhibition of APJ may provide novel pharmacological interventions for neuropathic pain.

\section{Introduction}

The apelin receptor (APJ), a novel G-protein-coupled receptor, shares homology with the angiotensin II type 1 receptor and was considered an 'orphan' receptor prior to the discovery of apelin in $1998(1,2)$. Apelin is a product of the 77-amino acid precursor preproapelin, which yields numerous isoforms, including apelin-12, apelin-13, apelin-17 and apelin-36 $(3,4)$. Apelin-13 is composed of 13 amino acids at the C-terminus of preproapelin, and is highly conserved (5); it exhibits the strongest binding to APJ among all the isoforms (3). Following the pairing of apelin peptides with cognate ligands, they have been identified to serve various roles in physiological and pathophysiological states, including effects on the cardiovascular system (6-8), fluid equilibrium $(9,10)$, the adipoinsular signaling pathway $(11,12)$, the immune system (13) and neuroprotection (14).

The apelin-APJ system is located in the central and peripheral nervous systems $(3,15)$. In the central nervous system (CNS), apelin and its receptors were detected in pain-associated regions, indicating that apelin may affect nociception $(4,16)$. Previous studies have indicated that apelin-13, when administered at the supraspinal level, inhibits acute or visceral pain (17,18). Intracerebroventricular apelin-13 administration promotes antinociception in mice, an effect markedly antagonized by coagulation factor XIII A chain (an APJ antagonist) and naloxone, suggesting that apelin is involved in opioid receptor signaling and corroborating reports that apelin is located in opioid-rich brain areas, including the arcuate nucleus of the hypothalamus and the spinal trigeminal nucleus (19). However, a previous study proposed that an intrathecal injection of apelin-13 produces hyperalgesia, not antinociception, in the second phase of a formalin test (20). The discrepancies between previous studies may result from 
the involvement of multiple receptor systems in apelin-13-induced pain behavior in acute and tonic pain models. Although apelin-13 produces aberrations in acute nociceptive models, it possibly serves an agonistic role at low concentrations, with these effects reversed at increased concentrations (20). Alternatively, apelin-13 may costimulate receptors aside from known opioid binding molecules, compromising its antinociceptive activity (17). Recent clinical results also indicated that the ratio of apelin to the endothelin-1 precursor has a positive correlation with increased vaso-occlusive pain in pediatric patients with sickle cell disease (21), indicating that the role of the apelin-APJ system in pain modulation requires further research.

Pain following nerve injury, also termed neuropathic pain, remains a challenging field within basic and clinical science. The apelin-APJ system is involved in the modulation of nociception, and in inflammatory and visceral pain; however, its involvement in models of neuropathic pain remain unclear. In addition, the signal transduction pathways of apelin overlap with those associated with neuropathic pain (22-24). Therefore, the present study was designed to examine the expression of the spinal apelin-APJ system and its functional role, and the mechanisms underlying neuropathic pain caused by chronic constriction injury (CCI) to the peripheral sciatic nerve.

\section{Materials and methods}

Animal preparation. Adult male Sprague-Dawley rats ( $\mathrm{n}=163 ;$ 200-220 g; 8-10-weeks-old) were purchased from the Experimental Animal Center of Guangdong Province (Guangdong, China). Rats were housed in a temperature-controlled room $\left(24 \pm 2^{\circ} \mathrm{C}\right)$ with a $12 \mathrm{~h}$ light/dark cycle and food and water available ad libitum. The animals were allowed to acclimate for $\geq 3$ days, prior to the experiments approved by the Animal Use and Care Committee for Research and Education of The First People's Hospital of Foshan (Guangdong, China), in accordance with the ethical guidelines of the International Association for the Study of Pain (25). Pain and suffering were minimized, as was the number of animals used.

CCI model. The CCI neuropathic pain model was established as previously proposed (26). The animals were anesthetized by intraperitoneal injection of $10 \%$ chloral hydrate $(400 \mathrm{mg} / \mathrm{kg})$. The biceps femoris was dissected to expose the left sciatic nerve. The area proximal to the sciatic nerve's trifurcation was freed of adhering tissue, and 4-0 surgical catgut suture was tied loosely around it with approximately $1 \mathrm{~mm}$ between ligatures. Sham-operated rats underwent the same surgery, but without ligation. Following the surgery, muscle and skin tissue was sutured in layers, and treatment with ampicillin $(100 \mathrm{mg} / \mathrm{kg})$ intraperitoneally for the first day was administered. Rats in the naïve group received neither surgery nor ligation. Each group contains 6 rats. Rats in the CCI group exhibiting no mechanical and thermal allodynia at 7 days post-surgery were excluded from the study.

Intrathecal treatment. In order to determine the effect of single intrathecal apelin-13 (10 $\mu \mathrm{g}$; Santa Cruz Biotechnology, Inc., Dallas, TX, USA) and ML221 (1, 3, 10 and $30 \mu \mathrm{g}$;
Sigma-Aldrich; Merck KGaA, Darmstadt, Germany) doses on mechanical allodynia and heat hyperalgesia, the rats were treated with light isoflurane anesthesia (1-2\%). The injection was carried out with a 30-gauge needle as previously described (27). Apelin-13 or ML221 was slowly administered in a solution of $10 \mu \mathrm{l}$.

A permanent intrathecal catheter (PE-10 polyethylene tube; BD Biosciences, Franklin Lakes, NJ) was inserted between the $T_{3}$ and $T_{4}$ vertebrae and extended to the $L_{4}$ and $\mathrm{L}_{5}$ segments following intraperitoneal administration of $400 \mathrm{mg} / \mathrm{kg}$ chloral hydrate, in order to assess the effect of continuous injection of apelin-13 or ML221 using behavioral testing. Cannulated animals were housed individually; those with surgery-associated neurological ailments were not taken into account in the final analysis. Drugs were injected through the catheter in a volume of $10 \mu \mathrm{l}$ followed by flushing with $10 \mu \mathrm{l}$ saline. All injections were performed by the same investigator.

Behavioral tests. Mechanical allodynia was evaluated as previously described (28). Each rat was placed individually into a wire mesh-bottomed cage for $30 \mathrm{~min}$ of adaptation prior to the test. Filaments were presented, from weakest to strongest, at a $90^{\circ}$ angle to the plantar surface, with enough force to slightly bend the paw, and maintained for $6 \mathrm{sec}$. A positive response was indicated by the animal quickly withdrawing or flinching the paw. The stimuli were presented at intervals of $2 \mathrm{~min}$, and the stimuli were increased and decreased sequentially ('up-and-down' technique) to assess paw-withdrawal threshold (PWT). Interpolation of the 50\% threshold (the weakest force causing $\geq 3$ withdrawals in 5 successive applications) was carried out according to the method of Dixon (29). The experiments were performed in a blinded manner.

Paw-withdrawal latency (PWL) values following thermal hyperalgesia were determined using a Plantar Analgesia Meter (IITC Life Science, Woodland Hills, CA, USA) (30). Individual rats were assessed three times (left hind paw) and average values were obtained.

Reverse transcription-quantitative polymerase chain reaction (RT-qPCR). The total RNA was obtained from $\mathrm{L}_{4}-\mathrm{L}_{5}$ spinal cord segments using TRIzol reagent (Invitrogen; Thermo Fisher Scientific, Inc., Waltham, MA, USA) according to the manufacturer's protocol. RNA purity and concentration were evaluated using the NanoDrop 2000 (Thermo Fisher Scientific, Inc., Wilmington, DE, USA). RT-qPCR analysis was carried out to determine the mRNA concentration of apelin and APJ using the EXPRESS One-Step SYBR Green ER SuperMix kit (Invitrogen; Thermo Fisher Scientific, Inc.) following the manufacturer's protocol, on a StepOne Real-Time PCR System (Applied Biosystems; Thermo Fisher Scientific, Inc.). Relative gene expression was assessed by the $2^{-\Delta \Delta} \mathrm{Cq}$ method (31). RT-qPCR amplification was carried out as previously described (30), with $\beta$-actin as the reference gene for normalization. The experiments were performed in triplicate. The specific primers used for sequence detection were as follows: $\beta$-actin, forward 5'-GGAGATTACTGCCCT GGCTCCTA-3' and reverse 5'-GACTCATCGTACTCCTGC TTGCTG-3'; Apelin, forward 5'-CTCTCCTTGACTGCCGTG TGTG-3' and reverse 5'-GCATGTTGCCTTCTTCTAGCC 
C-3'; APJ, forward 5'-TGCCTCAACCCCTTCCTCTA-3' and reverse 5'-GTTCTCCTCCCTTGCACATG-3'.

Western blotting. In order to evaluate the variation in the protein concentrations of the apelin-APJ system (rats randomly divided into control group, sham group and CCI groups) and ERK signaling [rats randomly divided into sham group, CCI group, CCI + dimethylsufoxide (DMSO) group and CCI + ML221 group], western blot analysis was performed with $\mathrm{L}_{4}-\mathrm{L}_{5}$ spinal cord segment specimens. Sample homogenization was performed in lysis buffer as described previously (30). Protein amounts were determined using the bicinchoninic acid assay. Equal amounts $(20 \mu \mathrm{g})$ of total protein were resolved by $10 \%$ SDS-PAGE and electro-transferred onto polyvinylidene fluoride membranes (Merck $\mathrm{KGaA}$ ). The membranes were blocked using $5 \%$ nonfat dry milk for $1 \mathrm{~h}$ at room temperature $\left(25^{\circ} \mathrm{C}\right)$ and incubated overnight at $4^{\circ} \mathrm{C}$ with primary antibodies raised against apelin (goat; cat. no. sc-33469; 1:1,500), APJ (rabbit; cat. no. sc-33823; 1:2,000), ERK1/2 (mouse; cat. no. sc-514302; 1:1,500), and p-ERK1/2 (rabbit; cat. no. sc-101760; 1:1,000), all purchased from Santa Cruz Biotechnology, Inc. For loading control, the blots were probed with GAPDH antibody (mouse; cat. no. sc-32233; 1:5,000; Santa Cruz Biotechnology, Inc.). These membranes were further incubated for $2 \mathrm{~h}$ with rabbit anti-goat (cat. no. BA1060; 1:2,000; Wuhan Boster Biological Technology, Ltd., Wuhan, China), goat anti-mouse (cat. no. BA1051; 1:2,000; Wuhan Boster Biological Technology, Ltd.) and mouse anti-rabbit (cat. no. L27A9; 1:2,000; Cell Signaling Technology, Inc., Danvers, MA, USA) IgG horseradish peroxidase-conjugated secondary antibodies at room temperature. Band intensities were assessed using Image $\mathrm{J}$ software (version $1.48 \mathrm{u}$; National Institutes of Health, Bethesda, MD, USA).

Immunohistochemistry. When neuropathic pain was fully established, the rats were intraperitoneally administered $400 \mathrm{mg} / \mathrm{kg}$ chloral hydrate for deep anesthesia, and $\mathrm{L}_{4}-\mathrm{L}_{5}$ spinal cord segments were extracted, fixed in $4 \%$ paraformaldehyde for $90 \mathrm{~min}$ at room temperature $\left(25^{\circ} \mathrm{C}\right)$ and then placed in $30 \%$ sucrose solution at $4^{\circ} \mathrm{C}$ overnight, embedded in optimal cutting temperature (OCT) compound (Thermo Fisher Scientific, Inc.), and sectioned at $5 \mu \mathrm{m}$ for immunofluorescence. Blocking with $2 \%$ donkey serum (Jackson ImmunoResearch Laboratories, Inc., West Grove, PA, USA) for $1 \mathrm{~h}$ at room temperature was followed by overnight incubation $\left(4^{\circ} \mathrm{C}\right)$ with primary antibodies raised against apelin (goat; cat. no. sc-33469; 1:100; Santa Cruz Biotechnology, Inc.) and APJ (rabbit; cat. no. sc-33823; 1:100; Santa Cruz Biotechnology, Inc.). DyLight 649- (rabbit anti-goat; cat. no. E032630; 1:200; EarthOx Life Sciences, Millbrae, CA, USA) or Andy Fluor 488-(goat anti-rabbit; cat. no. L110B; 1:300; GeneCopoeia, Inc., Rockville, MD, USA) conjugated secondary antibodies were added for $1 \mathrm{~h}$ at room temperature. Immunoreactivity was captured on a confocal laser scanning microscope (magnification, x200) and analyzed with Adobe Photoshop CS6 (Adobe Systems, Inc., San Jose, CA, USA).

Statistical analysis. All values are expressed as the mean \pm standard error of the mean. The data on mechanical allodynia and thermal hyperalgesia were analyzed by two-way repeated measures analysis of variance followed by the Bonferroni post hoc test. Western blotting and RT-qPCR data were analyzed by one-way analysis of variance followed by the Newman-Keuls post hoc test. Independent samples Student's t-test was used to analyze the immunofluorescence data. $\mathrm{P}<0.05$ was considered to indicate a statistically significant difference.

\section{Results}

Upregulated levels of the spinal apelin-APJ system are associated with CCI-induced neuropathic pain. The function of the apelin-APJ system in chronic neuropathic pain was investigated by examining apelin-APJ system expression at the mRNA and protein level in CCI rats. CCI caused rapid and persistent neuropathic pain from the third day following surgery, which lasted for $>2$ weeks in the rats. mRNA and protein levels were assessed at 3, 7 and 14 days post-injury; these time points corresponded, respectively, to the onset, peak and full establishment of allodynia post-CCI. RT-qPCR data demonstrated that apelin mRNA levels continuously increased, peaking at 7 days post-CCI, with a 3-fold increase in the CCI group compared with the controls (Fig. 1A). In addition, a parallel, significant and time-dependent increase in APJ mRNA levels, was observed at 3, 7 and 14 days post-CCI (Fig. 1B). Western blot analysis demonstrated that CCI caused a rapid (within 3 days) and long-lasting (>14 days) increase in APJ protein amounts in the spinal cord (Fig. 2B). Additionally, spinal apelin protein levels were significantly increased, compared with sham-operated animals, 7 and 14 days post-surgery (Fig. 2A).

In order to examine spinal cord apelin and APJ expression levels, immunostaining was carried out in naïve and CCI-operated mice. As the expression levels of apelin and APJ peaked at 7 days post-surgery, this time point was selected to assess the alteration of apelin and APJ expression levels in the spinal cord. As presented in Fig. 3B, APJ was constitutively expressed in the superficial dorsal horn. CCI induced a marked increase in APJ expression and an increased number of APJ-immunoreactive cells was observed in the CCI group compared with the sham group (Fig. 3B). In addition, low immunofluorescence intensities were obtained for apelin in sham animals, while CCI rats exhibited enhanced expression 7 days post-surgery (Fig. 3A).

The results of the present study suggested that pain-associated alterations of apelin and APJ levels in the superficial dorsal horn may be involved in the development and maintenance of chronic neuropathic pain.

Spinal apelin-APJ system affects neuropathic pain development and maintenance. Mechanical allodynia and thermal hyperalgesia in the animals were measured at 1 day before (baseline), and 1, 3, 5 and 7 days following, CCI or sham surgery. It was identified that CCI led to a gradual reduction in PWT and PWL to a level considered to be consistent with neuropathic pain, in accordance with a previous report (26).

In order to investigate the role of increased expression of the spinal apelin-APJ system in pain sensory modulation, intrathecal injections of apelin-13 and ML221 were performed in CCI rats. A single intrathecal injection of $10 \mu \mathrm{g}$ ML221, 7 days following CCI, caused a transient, marked reduction of mechanical allodynia and heat hyperalgesia compared with CCI rats treated with normal saline (Fig. 4A and B). By 
A

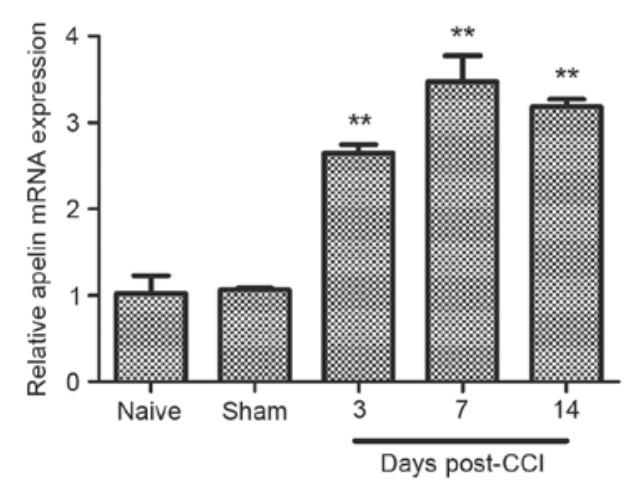

B

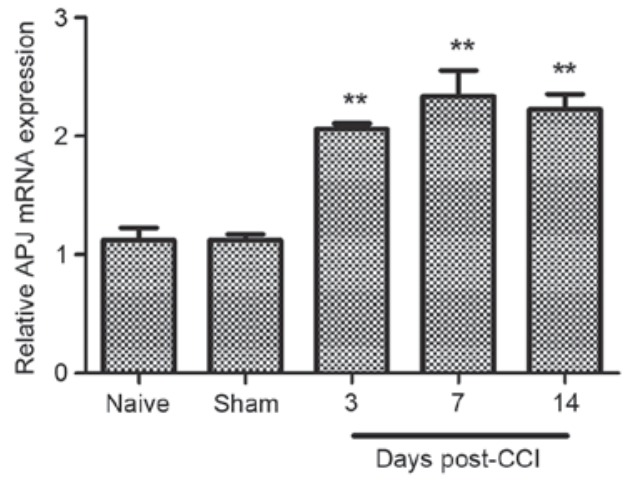

Figure 1. Expression of apelin and APJ mRNA in rat spinal cord following CCI. Reverse transcription-quantitative polymerase chain reaction analysis presenting the time course for (A) apelin and (B) APJ mRNA expression in the spinal cord ( $\mathrm{n}=6$ in each case). Data demonstrated that apelin and APJ mRNA levels increased at 3,7 and 14 days post-CCI compared with the sham group. No obvious difference was observed between naïve and sham groups. mRNA levels were normalized to $\beta$-actin. Data are presented as the mean \pm standard error of the mean. Data were analyzed using one-way analysis of variance. ${ }^{* *} \mathrm{P}<0.01$ vs. sham group. APJ, apelin receptor; $\mathrm{CCI}$, chronic constriction injury.

A
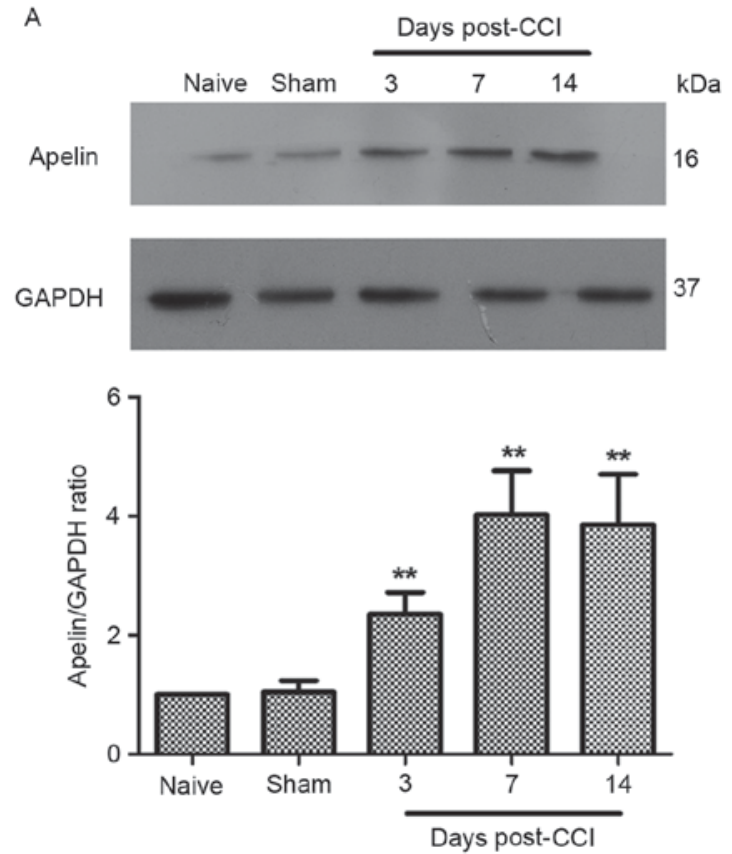

B
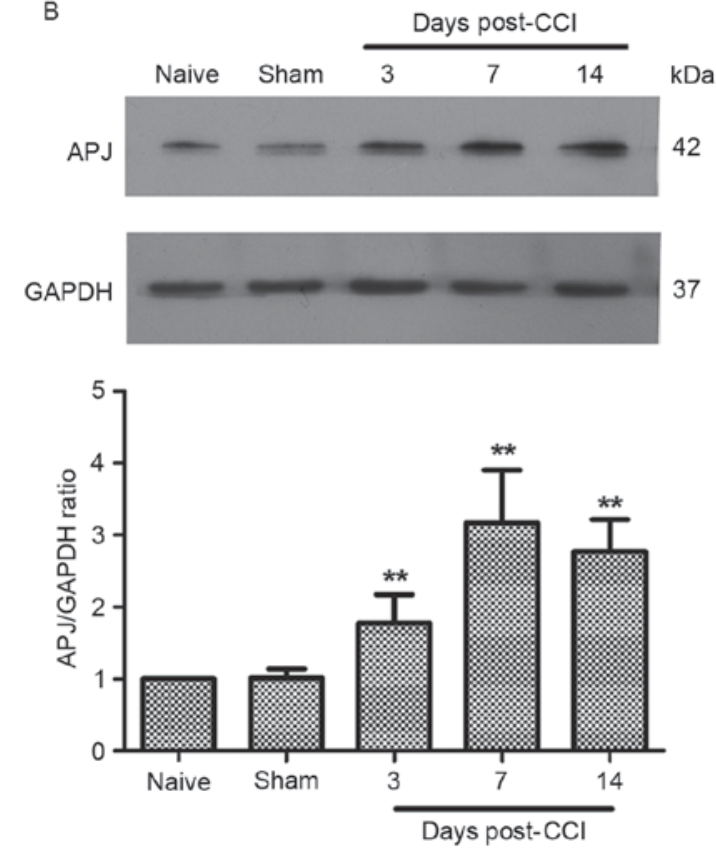

Figure 2. Expression of apelin and APJ protein in rat spinal cord following CCI. Western blot analysis presenting the time course for (A) apelin and (B) APJ protein expression in the spinal cord ( $\mathrm{n}=6$ in each case). The left sciatic nerve of the rats in CCI group was exposed and tied loosely by 4-0 surgical catgut. Sham group underwent the same surgery, but without ligation. Rats in naïve group received neither surgery nor ligation. Results demonstrated that apelin and APJ protein levels increased at 3,7 and 14 days post-CCI compared with the sham group. There were no obvious differences between naïve and sham groups. GAPDH was used as an internal standard. Data are presented as the mean \pm standard error of the mean. Data were analyzed using one-way analysis of variance. ${ }^{* *} \mathrm{P}<0.01$ vs. sham group. APJ, apelin receptor; $\mathrm{CCI}$, chronic constriction injury.

contrast, an intrathecal injection of apelin-13 did not affect mechanical allodynia or heat hyperalgesia (Fig. 4A and B), suggesting that the spinal apelin-APJ system contributed to CCI-induced neuropathic pain. Drug dosages were selected based on a previous study (20).

In order to study the dose-effect association of ML221 in alleviating CCI-induced neuropathic pain, increasing doses of ML221 (1, 3, 10 and $30 \mu \mathrm{g})$ were injected intrathecally 7 days post-surgery. As presented in Fig. 4C and D, ML221 inhibited mechanical allodynia and thermal hyperalgesia in a dose-dependent manner; these effects began $1 \mathrm{~h}$ post-injection, peaked at $2 \mathrm{~h}$ and diminished within $4 \mathrm{~h}$. A dose of $3 \mu \mathrm{g}$ ML221 transiently reduced CCI-induced mechanical allodynia and heat hyperalgesia, although the reduction was not statistically significant; this result was not observed with a $1 \mu \mathrm{g}$ dose. A $10 \mu \mathrm{g}$ dose of ML221 significantly increased PWT and PWL between 1 and $4 \mathrm{~h}$. Increased ML221 dosages displayed analgesic effects similar to those obtained with $10 \mu \mathrm{g}$, indicating that a single intrathecal injection of $10 \mu \mathrm{g}$ ML221 may be considered the optimal dose for the reversal of allodynia.

In order to assess the long-term impact of chronic intrathecal ML221 administration during the initiation of CCI-induced neuropathic pain, $10 \mu \mathrm{g}$ ML221 was administered once daily for 3 days (days 0-2). Pretreatment with ML221 for 

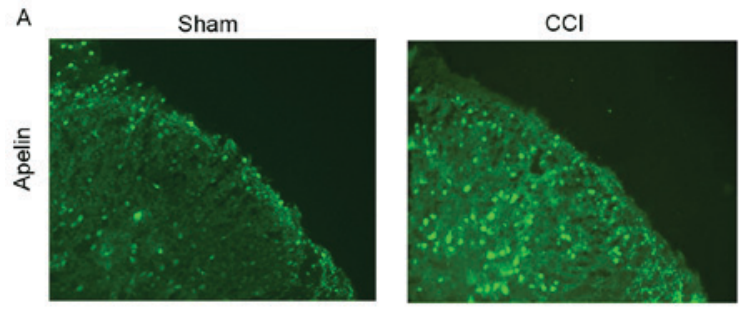

B
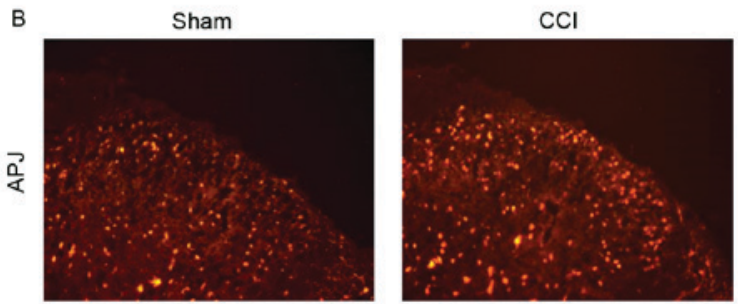
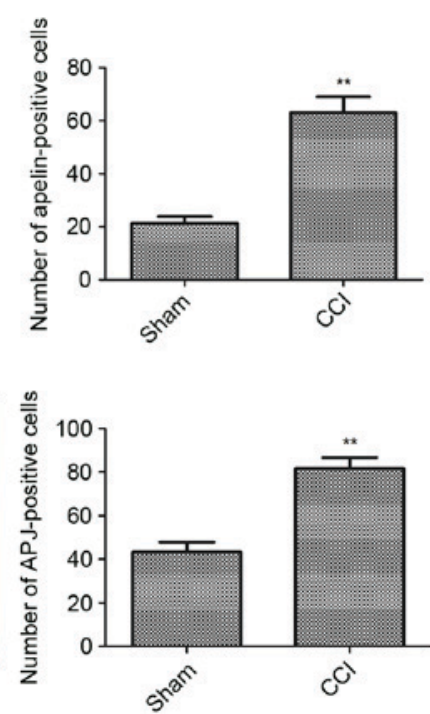

Figure 3. Immunofluorescence demonstrating the expression and distribution of apelin (green) and APJ (red) in the superficial dorsal horn ipsilateral to the CCI. The (A) apelin and (B) APJ expression in the CCI group was increased compared with the sham group. Samples for immunofluorescence were collected at 7 days post-injury. Original magnification, x200. Scale bar, $50 \mu \mathrm{m}$. Data are presented as the mean \pm standard error of the mean. Data were analyzed using a t-test. ${ }^{* *} \mathrm{P}<0.01$ vs. sham group. APJ, apelin receptor; $\mathrm{CCI}$, chronic constriction injury.
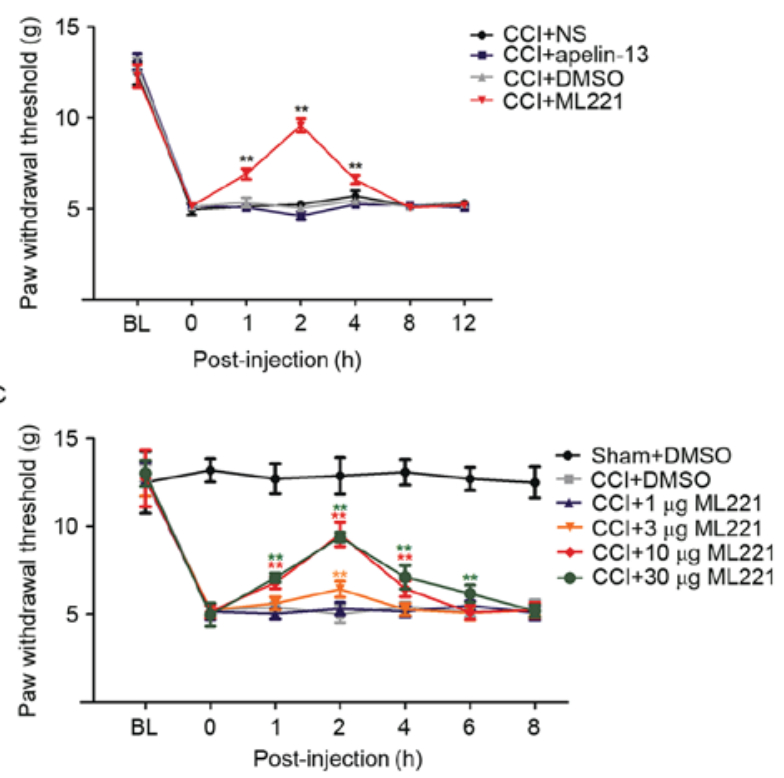

B

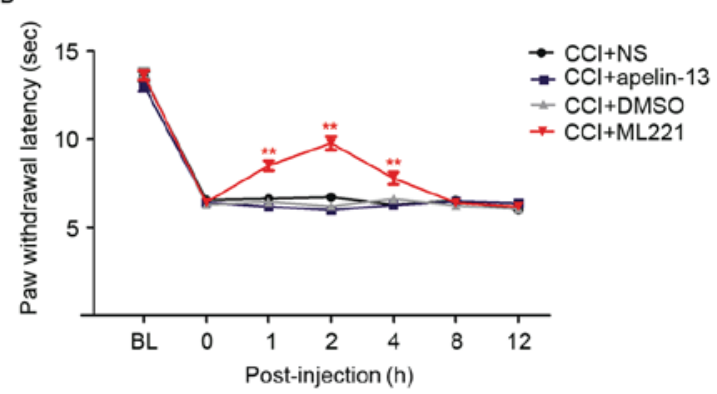

D

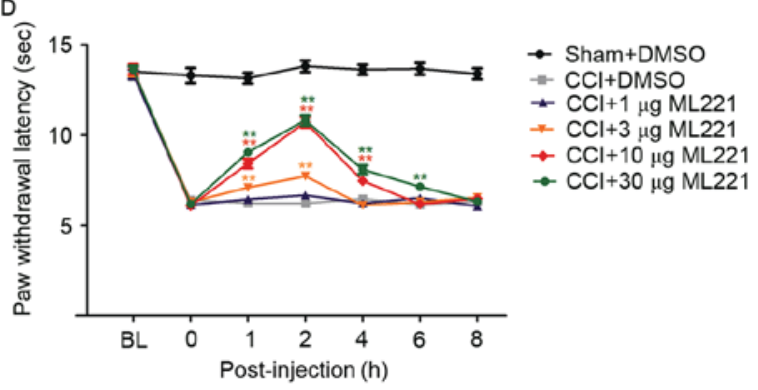

Figure 4. ML221 alleviates pain hypersensitivity induced by CCI of the sciatic nerve. The time course of the effects of intrathecal injection of apelin-13 $(10 \mu \mathrm{g})$ and ML221 (10 $\mu \mathrm{g})$ on (A) mechanical allodynia and (B) thermal hyperalgesia at 7 days post-CCI. Behavioral tests were performed before CCI surgery (baseline) and prior to ( $0 \mathrm{~h}$ ) and 1, 2, 4, 8 and $12 \mathrm{~h}$ following ML221 administration. Increasing doses of ML221 (1, 3, 10 and 30 $\mu \mathrm{g})$ were administered at 7 days post-CCI. (C) Paw withdrawal time and (D) paw withdrawal latency were measured prior to CCI surgery (baseline) and prior to (0 h) and $1,2,4,6$ and $8 \mathrm{~h}$ following ML221 administration. A total of eight rats were included in each group. Data are presented as the mean \pm standard error of the mean. Data were analyzed using two-way analysis of variance. ${ }^{* *} \mathrm{P}<0.01$ vs. CCI + DMSO group. CCI, chronic constriction injury; DMSO, dimethyl sulfoxide.

3 successive days markedly delayed CCI-induced mechanical allodynia and heat hyperalgesia for 2-11 days (Fig. 5A and B). In order to determine the long-term effect of repeated treatment with ML221 in maintaining CCI-induced neuropathic pain, the same ML221 doses were given at days 7-9, and produced a somewhat reliable inhibitory effect on allodynia (Fig. 5C and D). Analgesia persisted for 4 days following the third treatment with ML221. The present results suggested that a reduction in the expression of spinal APJ may inhibit the development and maintenance of CCI-induced neuropathic pain.

Alleviation of CCI-induced neuropathic pain by ML221 is associated with phosphorylated ERK downregulation in the spinal cord. As presented above, increased expression of the spinal apelin-APJ system contributes to CCI-induced neuropathic pain. As ERK signaling is implicated in the effects of apelin, the present study investigated whether apelin-induced 

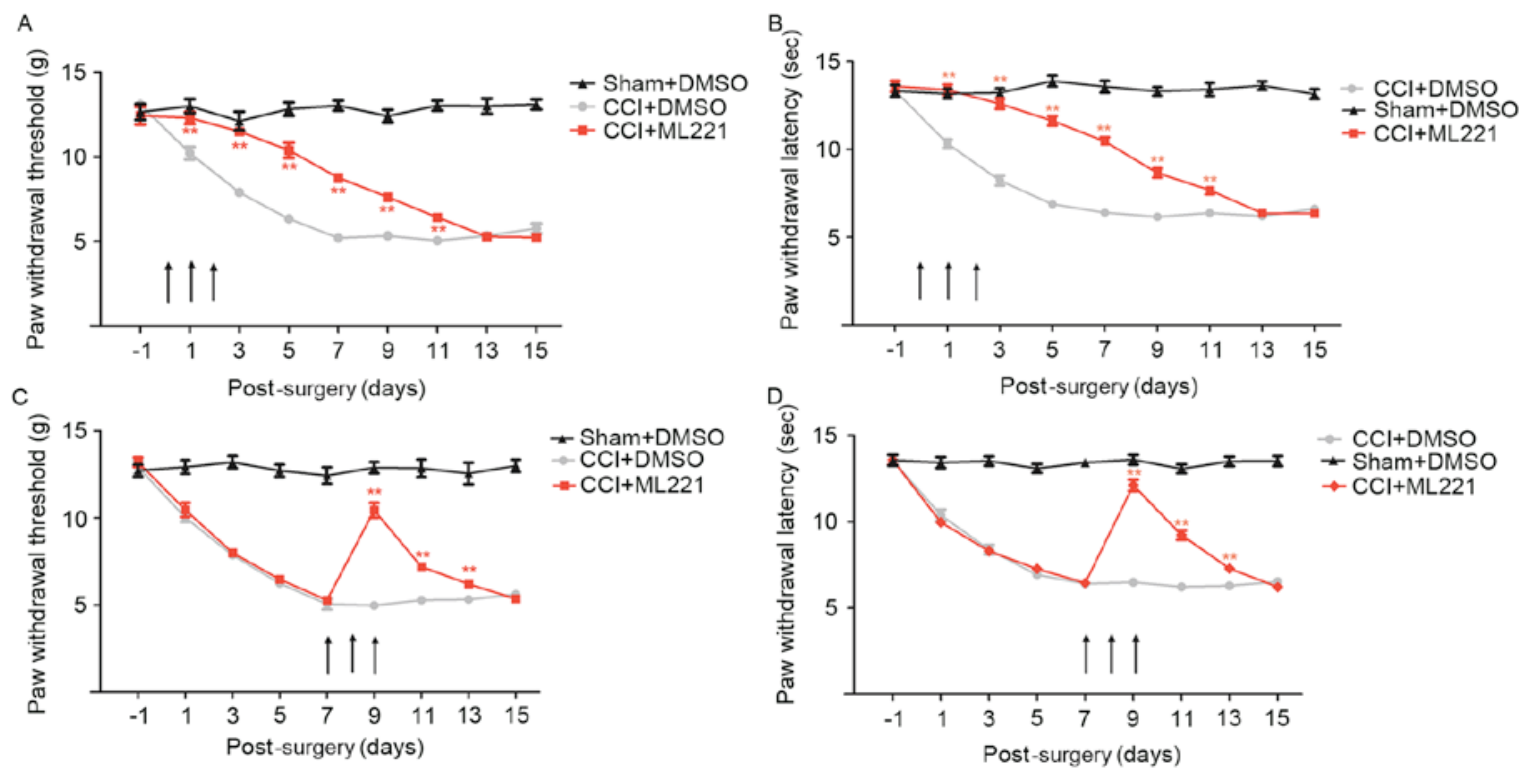

Figure 5. Repeated intrathecal injections of ML221 delays the development and partially reverses the maintenance of CCI-induced neuropathic pain. Repeated administration of ML221 (10 $\mu \mathrm{g})$ for 3 consecutive days (day 0-2) in the early phase delayed the onset of (A) mechanical allodynia and (B) thermal hyperalgesia. Behavioral tests were performed 1 day prior to CCI surgery and 1,3,5, 7,9,11,13 and 15 days post-surgery. Repeated injections of ML221 (10 $\mu \mathrm{g}$ ) for 3 consecutive days (day 7-9) in the later phase significantly alleviated CCI-induced (C) mechanical allodynia and (D) thermal hyperalgesia. A total of eight rats were included in each group. Data are presented as the mean \pm standard error of the mean. Data were analyzed using two-way analysis of variance. ${ }^{* *} \mathrm{P}<0.01$ vs. CCI + DMSO group. CCI, chronic constriction injury; DMSO, dimethyl sulfoxide.

nociceptive behaviors were mediated via the ERK signaling pathway. Rats were assigned to sham, CCI, CCI + DMSO, and CCI + ML221 groups, respectively. A single intrathecal injection of ML221 (10 $\mu \mathrm{g})$ or vehicle (DMSO) was performed 7 days post-surgery, and $\mathrm{L}_{4}-\mathrm{L}_{5} \mathrm{SC}$ segments were harvested $2 \mathrm{~h}$ post-injection for the assessment of ERK and phosphorylated ERK levels. As presented in Fig. 6, phosphorylated ERK levels were increased in the CCI group compared with the sham group. Phosphorylated ERK induction was alleviated by a single intrathecal injection of ML221, corroborating the results obtained in the behavioral tests as described above. The present data indicated that the spinal apelin-APJ system may be involved in neuropathic pain via the ERK signaling pathway.

\section{Discussion}

Current evidence suggests that apelin serves a role in neuronal signaling pathways. In a previous study, apelin was not detected in glial cells in clinical and rat epilepsy models (32). In mice with amyotrophic lateral sclerosis, spinal cord apelin levels are significantly decreased due to apelin silencing (33). There is increasing evidence to suggest an association of apelin with inflammatory processes, as inflammatory mediators, including tumor necrosis factor- $\alpha$ (34), interleukin- 6 , and interferon- $\gamma(35)$ may increase apelin expression levels, which correlate with markers of inflammation (36). In addition, apelin was suggested to be involved in pain modulation.

The present study provides an insight into the function of apelin in neuropathic pain. It was demonstrated that the spinal apelin-APJ system serves a role in the modulation of nerve injury-induced neuropathic pain. Apelin and APJ expression levels were increased in the ipsilateral spinal dorsal horn following CCI. Spinal blockade does not activate APJ; rather,
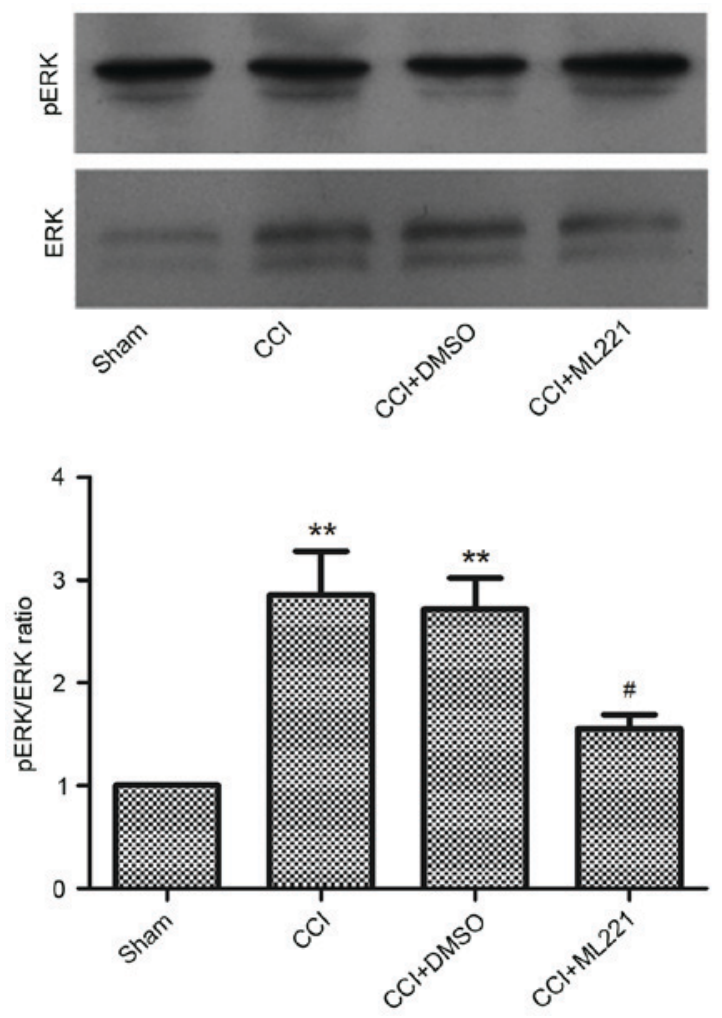

Figure 6. ML221 inhibits the CCI-induced activation of ERK in the rat spinal cord. The representative immunoblotting bands and the quantitative data demonstrated that ML221 inhibited the CCI-induced increase of pERK expression in the spinal cord. ML221 $(10 \mu \mathrm{g})$ was intrathecally injected at 7 days post-CCI. $\mathrm{L}_{4}-\mathrm{L}_{5}$ spinal cord segments were collected in order to measure ERK and pERK expression at $2 \mathrm{~h}$ following ML221 injection ( $\mathrm{n}=6$ in each case). There was no obvious change between sham group and CCI group. Data are presented as the mean \pm standard error of the mean. Data were analyzed using one-way analysis of variance. ${ }^{* *} \mathrm{P}<0.01 \mathrm{vs}$. sham group, ${ }^{\text {"}} \mathrm{P}<0.01$ vs. CCI group. CCI, chronic constriction injury; pERK, phosphorylated extracellular signal-related kinase 
it inhibits CCI-associated neuropathic pain development and persistence. The apelin-APJ system may contribute to neuropathic pain via ERK signaling by upregulating phosphorylated ERK in the spinal dorsal horn. Therefore, the apelin-APJ system may be a novel mechanism and target in neuropathic pain.

Apelin-13 is the strongest APJ activator expressed in cells $(3,15)$. As an adipocytokine, apelin is expressed in and secreted by mature mammalian adipocytes (37). Apelin-13 is also highly expressed in parts of the CNS, including the hypothalamus, hippocampus, striatum, pituitary gland, substantia nigra, central gray matter, dorsal raphe nucleus, amygdala, cerebellum and spinal cord (19,38-42). In the present study, a long-lasting increase in apelin mRNA and protein expression levels in the spinal cord following nerve injury was observed, as were persistent pain behaviors. Immunofluorescent staining demonstrated that apelin was expressed in the superficial spinal dorsal horn. APJ is widely distributed in the CNS and periphery; the overlap in receptor and peptide expression patterns demonstrates the variety of neuronal functions of APJ. The present data indicated that peripheral nerve injury upregulated APJ mRNA and protein expression from day 3 , peaking at day 7 , in CCI rats. Increased APJ immunoreactive signals were observed in the ipsilateral spinal cord dorsal horn. The present data demonstrate that the spinal apelin-APJ system is activated during nerve injury, and therefore may be implicated in pain processes.

Previous studies have reported various effects of the apelin-APJ system in pain modulation, and suggested that the differences may be due to apelin-13-induced pain behavior involving distinct receptor systems in acute and tonic pain models $(17,18,20)$. In order to further clarify the function of the spinal apelin-APJ system in neuropathic pain, apelin-13 and ML221 were administered intrathecally to CCI rats. As presented above, a single intrathecal injection of $10 \mu \mathrm{g}$ apelin-13 exhibited no effect on pain behavior; however, ML221, a newly-synthesized APJ antagonist, significantly reduced CCI-induced pain hypersensitivity. Nerve injury increased apelin expression and the APJ antagonist ML221 alleviated CCI-induced neuropathic pain, indirectly indicating a role for endogenous apelin in neuropathic pain development, consistent with previous formalin test data (20). In the present study, ML221 was used as it inhibits apelin-13-mediated recruitment of $\beta$-arrestin and exhibits 37 -fold increased selectivity for APJ compared with the angiotensin II type 1 receptor (43). The dose-effect association of ML221 in neuropathic pain was investigated. Increasing ML221 doses (1, 3, 10 and $30 \mu \mathrm{g}$ ) were administered intrathecally at day 7 to CCI rats, and 3, 10 and $30 \mu \mathrm{g}$ partially reversed mechanical allodynia and heat hyperalgesia. Notably, ML221 at 10 and $30 \mu \mathrm{g}$ exhibited similar pharmacological effects in relieving CCI-induced hyperalgesia, indicating that ML221 had reached peak efficacy. Neuropathic pain involves abnormal production, distribution and activity of multiple receptors, ion channels and signaling pathway effectors; therefore, it is understandable that the inhibition of APJ may not fully reverse pain behaviors. In a preventive approach, intrathecal administration of $10 \mu \mathrm{g}$ ML221 at days 0-2 prevented mechanical allodynia and markedly reduced the thermal hyperalgesia which ordinarily occurs 3 days following CCI. Intrathecal administration of $10 \mu \mathrm{g}$
ML221 at days 7-9, when thermal and mechanical hyperalgesia reached full development, attenuated thermal hyperalgesia and mechanical allodynia at days 7,9 and 11. The results of the present study suggested a role for apelin in the initiation and persistence of neuropathic pain.

The role of apelin in the modulation of pain remains unclear. Previous evidence indicates that there is functional cross-talk between APJ and opioid receptors. In a previous study, apelin mRNA expression was decreased in the lateral hypothalamus of morphine-dependent mice (44); in addition, the inhibitory effects of apelin-13 on gastrointestinal motility involve opioid receptors (45). $\mu$-opioid receptors were suggested to mediate the antinociceptive activity of apelin-13, which has been demonstrated to markedly amplify the analgesic effect of morphine in a tail immersion test and a visceral pain model $(17,18)$. Spinal apelin-13 has been observed to cause hyperalgesia in a tonic inflammatory pain model, an effect mediated by APJ and the $\gamma$-aminobutyric acid receptor A, not opioid receptors (20). Similar phenomena involving interactions between distinct receptor systems have been reported previously (46). In the present study, intrathecal injection of $10 \mu \mathrm{g}$ apelin-13, an increased dose compared with that which has been demonstrated to induce a significant antinociceptive effect in a tail-flick test (20), exhibited no impact on pain sensitivity, while inhibition of APJ significantly reduced mechanical allodynia and heat hyperalgesia, indicating that increased apelin and APJ expression levels contribute to CCI-induced neuropathic pain.

Previous studies in the field of pain research have focused on the effects of opioid peptides in apelin signaling, and less research has been focused on the role of apelin, particularly in chronic pain. APJ possesses a typical 7-transmembrane domain structure with consensus phosphorylation sites (1). The activation of apelin receptors inhibits forskolin-stimulated cyclic AMP synthesis (47). In addition to the adenylyl cyclase inhibition pathway, in vitro studies have demonstrated that apelin is involved in ERK1/2 induction in a protein kinase C-dependent pathway (48). Apelin-13 and apelin-36 have been observed to promote ERK1/2 phosphorylation in Chinese hamster ovary cells and hippocampal cultures stably producing murine APJ $(49,50)$. A comparable previous study investigating HEK293 cells transfected with human APJ also identified ERK1/2 induction by apelin (51). Apelin, acting via APJ, may activate multiple signaling pathways. The transcription factors or other cell mediators involved in the action of apelin in CCI-induced neuropathic pain remain unclear. It is known that ERK induction in spinal cord neurons through nociceptive activity, involving various neurotransmitters and relay molecules, is involved in CNS sensitization and pain hypersensitivity, regulating the activities of multiple ligands and increasing mRNA synthesis (52). The present study investigated whether the hyperalgesic effect of the spinal apelin-APJ system on neuropathic pain was mediated through ERK signaling. As presented above, intrathecal ML221 administration downregulated phosphorylated ERK and alleviated pain behaviors. Therefore, ERK phosphorylation and activation may be a downstream effect of apelin signaling which drives CCI-induced neuropathic pain. However, future investigation of the function of apelin, in numerous aspects of neuropathic pain, is required to clarify the underlying mechanisms. 
Clinical approaches to treating neuropathic pain are scarce, and the mechanisms behind neuropathic pain are not completely understood; thus, neurochemical alterations accompanying neuropathic pain may be considered targets for its treatment. The present study describes the role of spinal apelin in the processing of neuropathic pain induced by chronic constriction of the sciatic nerve, and elucidates receptor and intracellular mechanisms underlying apelin-mediated pain modulation. The results of the present study provide a basis for the development of novel therapeutics for chronic neuropathic pain.

\section{References}

1. O'Dowd BF, Heiber M, Chan A, Heng HH, Tsui LC, Kennedy JL, Shi X, Petronis A, George SR and Nguyen T: A human gene that shows identity with the gene encoding the angiotensin receptor is located on chromosome 11. Gene 136: 355-360, 1993.

2. Tatemoto K, Hosoya M, Habata Y, Fujii R, Kakegawa T, Zou MX, Kawamata Y, Fukusumi S, Hinuma S, Kitada C, et al: Isolation and characterization of a novel endogenous peptide ligand for the human APJ receptor. Biochem Biophys Res Commun 251: 471-476, 1998.

3. Kawamata Y, Habata Y, Fukusumi S, Hosoya M, Fujii R, Hinuma S, Nishizawa N, Kitada C, Onda H, Nishimura O and Fujino M: Molecular properties of apelin: Tissue distribution and receptor binding. Biochim Biophys Acta 1538: 162-171, 2001

4. Hosoya M, Kawamata Y, Fukusumi S, Fujii R, Habata Y, Hinuma S, Kitada C, Honda S, Kurokawa T, Onda H, et al: Molecular and functional characteristics of APJ. Tissue distribution of mRNA and interaction with the endogenous ligand apelin. J Biol Chem 275: 21061-21067, 2000.

5. Lee HJ, Tomioka M, Takaki Y, Masumoto H and Saido TC: Molecular cloning and expression of aminopeptidase A isoforms from rat hippocampus. Biochim Biophys Acta 1493: 273-278, 2000.

6. Japp AG and Newby DE: The apelin-APJ system in heart failure: Pathophysiologic relevance and therapeutic potential. Biochem Pharmacol 75: 1882-1892, 2008.

7. Chandrasekaran B, Dar O and McDonagh T: The role of apelin in cardiovascular function and heart failure. Eur J Heart Fail 10: 725-732, 2008

8. Quazi R, Palaniswamy C and Frishman WH: The emerging role of apelin in cardiovascular disease and health. Cardiol Rev 17: 283-286, 2009

9. Brown $\mathrm{CH}$, Ruan M, Scott V, Tobin VA and Ludwig M: Multi-factorial somato-dendritic regulation of phasic spike discharge in vasopressin neurons. Prog Brain Res 170: 219-228, 2008.

10. Llorens-Cortes C and Moos F: Opposite potentiality of hypothalamic coexpressed neuropeptides, apelin and vasopressin in maintaining body-fluid homeostasis. Prog Brain Res 170 559-570, 2008

11. Rayalam S, Della-Fera MA, Krieg PA, Cox CM, Robins A and Baile CA: A putative role for apelin in the etiology of obesity. Biochem Biophys Res Commun 368: 815-819, 2008.

12. Lee DK, George SR and O'Dowd BF: Unravelling the roles of the apelin system: Prospective therapeutic applications in heart failure and obesity. Trends Pharmacol Sci 27: 190-194, 2006.

13. Horiuchi Y, Fujii T, Kamimura Y and Kawashima K: The endogenous, immunologically active peptide apelin inhibits lymphocytic cholinergic activity during immunological responses. J Neuroimmunol 144: 46-52, 2003.

14. Cheng B, Chen J, Bai B and Xin Q: Neuroprotection of apelin and its signaling pathway. Peptides 37: 171-173, 2012.

15. Medhurst AD, Jennings CA, Robbins MJ, Davis RP, Ellis C, Winborn KY, Lawrie KW, Hervieu G, Riley G, Bolaky JE, et al: Pharmacological and immunohistochemical characterization of the APJ receptor and its endogenous ligand apelin. J Neurochem 84: 1162-1172, 2003.

16. Reaux A, De Mota N, Skultetyova I, Lenkei Z, El Messari S, Gallatz K, Corvol P, Palkovits M and Llorens-Cortes C: Physiological role of a novel neuropeptide, apelin, and its receptor in the rat brain. J Neurochem 77: 1085-1096, 2001.

17. Xu N, Wang H, Fan L and Chen Q: Supraspinal administration of apelin-13 induces antinociception via the opioid receptor in mice. Peptides 30: 1153-1157, 2009.
18. Lv SY, Qin YJ, Wang NB, Yang YJ and Chen Q: Supraspinal antinociceptive effect of apelin-13 in a mouse visceral pain model. Peptides 37: 165-170, 2012.

19. Reaux A, Gallatz K, Palkovits $M$ and Llorens-Cortes C: Distribution of apelin-synthesizing neurons in the adult rat brain. Neuroscience 113: 653-662, 2002.

20. Lv S, Yang YJ, Hong S, Wang N, Qin Y, Li W and Chen Q: Intrathecal apelin-13 produced different actions in formalin test and tail-flick test in mice. Protein Pept Lett 20: 926-931, 2013.

21. Smith TP, Schlenz AM, Schatz JC, Maitra R and Sweitzer SM: Modulation of pain in pediatric sickle cell disease: Understanding the balance between endothelin mediated vasoconstriction and apelin mediated vasodilation. Blood Cells Mol Dis 54: 155-159, 2015.

22. Cui RR, Mao DA, Yi L, Wang C, Zhang XX, Xie H, Wu XP, Liao XB, Zhou H, Meng JC, et al: Apelin suppresses apoptosis of human vascular smooth muscle cells via APJ/PI3-K/Akt signaling pathways. Amino Acids 39: 1193-1200, 2010.

23. Than A, Cheng Y, Foh LC, Leow MK, Lim SC, Chuah YJ, Kang Y and Chen P: Apelin inhibits adipogenesis and lipolysis through distinct molecular pathways. Mol Cell Endocrinol 362: 227-241, 2012.

24. Perjes A, Skoumal R, Tenhunen O, Kónyi A, Simon M, Horváth IG, Kerkelä R, Ruskoaho $\mathrm{H}$ and Szokodi I: Apelin increases cardiac contractility via protein kinase $\mathrm{C} \varepsilon$ - and extracellular signal-regulated kinase-dependent mechanisms. PLoS One 9: e93473, 2014

25. Zimmermann M: Ethical guidelines for investigations of experimental pain in conscious animals. Pain 16: 109-110, 1983.

26. Bennett GJ and Xie YK: A peripheral mononeuropathy in rat that produces disorders of pain sensation like those seen in man. Pain 33: 87-107, 1988

27. Mestre C, Pélissier T, Fialip J, Wilcox G and Eschalier A: A method to perform direct transcutaneous intrathecal injection in rats. J Pharmacol Toxicol Methods 32: 197-200, 1994

28. Chaplan SR, Bach FW, Pogrel JW, Chung JM and Yaksh TL: Quantitative assessment of tactile allodynia in the rat paw. J Neurosci Methods 53: 55-63, 1994.

29. Dixon WJ: Efficient analysis of experimental observations. Annu Rev Pharmacol Toxicol 20: 441-462, 1980.

30. Yang JX, Hua L, Li YQ, Jiang YY, Han D, Liu H, Tang QQ, Yang XN, Yin C, Hao LY, et al: Caveolin-1 in the anterior cingulate cortex modulates chronic neuropathic pain via regulation of NMDA receptor 2B subunit. J Neurosci 35: 36-52, 2015.

31. Livak KJ and Schmittgen TD: Analysis of relative gene expression data using real-time quantitative PCR and the 2(Delta Delta C(T)) method. Methods 25: 402-408, 2001.

32. Zhang X, Peng X, Fang M, Zhou C, Zhao F, Zhang Y, Xu Y, Zhu Q, Luo J, Chen G and Wang X: Up-regulation of apelin in brain tissue of patients with epilepsy and an epileptic rat model. Peptides 32: 1793-1799, 2011

33. Kasai A, Kinjo T, Ishihara R, Sakai I, Ishimaru Y, Yoshioka Y, Yamamuro A, Ishige K, Ito Y and Maeda S: Apelin deficiency accelerates the progression of amyotrophic lateral sclerosis. PloS One 6: e23968, 2011

34. Daviaud D, Boucher J, Gesta S, Dray C, Guigne C, Quilliot D, Ayav A, Ziegler O, Carpene C, Saulnier-Blache JS, et al: TNFalpha up-regulates apelin expression in human and mouse adipose tissue. FASEB J 20: 1528-1530, 2006.

35. Han S, Wang G, Qi X, Englander EW and Greeley GH Jr: Involvement of a Stat 3 binding site in inflammation-induced enteric apelin expression. Am J Physiol Gastrointest Liver Physiol 295: G1068-G1078, 2008.

36. Malyszko J, Malyszko JS, Pawlak K, Wolczynski S and Mysliwiec M: Apelin, a novel adipocytokine, in relation to endothelial function and inflammation in kidney allograft recipients. Transplant Proc 40: 3466-3469, 2008

37. Boucher J,Masri B, DaviaudD, Gesta S, Guigné C,Mazzucotelli A, Castan-Laurell I, Tack I, Knibiehler B, Carpéné C, et al: Apelin, a newly identified adipokine up-regulated by insulin and obesity. Endocrinology 146: 1764-1771, 2005.

38. Choe W, Albright A, Sulcove J, Jaffer S, Hesselgesser J, Lavi E, Crino P and Kolson DL: Functional expression of the seven-transmembrane HIV-1 co-receptor APJ in neural cells. J Neurovirol 6 (Suppl 1): S61-S69, 2000.

39. O'Carroll AM, Selby TL, Palkovits M and Lolait SJ: Distribution of mRNA encoding B78/apj, the rat homologue of the human APJ receptor, and its endogenous ligand apelin in brain and peripheral tissues. Biochim Biophys Acta 1492: 72-80, 2000.

40. Brailoiu GC, Dun SL, Yang J, Ohsawa M, Chang JK and Dun NJ: Apelin-immunoreactivity in the rat hypothalamus and pituitary. Neurosci Lett 327: 193-197, 2002. 
41. De Mota N, Lenkei Z and Llorens-Cortès C: Cloning, pharmacological characterization and brain distribution of the rat apelin receptor. Neuroendocrinology 72: 400-407, 2000.

42. Lee DK, Cheng R, Nguyen T, Fan T, Kariyawasam AP, Liu Y, Osmond DH, George SR and O'Dowd BF: Characterization of apelin, the ligand for the APJ receptor. J Neurochem 74: 34-41, 2000

43. Maloney PR, Khan P, Hedrick M, Gosalia P, Milewski M, Li L, Roth GP, Sergienko E, Suyama E, Sugarman E, et al: Discovery of 4-oxo-6-((pyrimidin-2-ylthio)methyl)-4H-pyran-3-yl 4-nitrobenzoate (ML221) as a functional antagonist of the apelin (APJ) receptor. Bioorg Med Chem Lett 22: 6656-6660, 2012.

44. Befort K, Filliol D, Darcq E, Ghate A, Matifas A, Lardenois A, Muller J, Thibault C, Dembele D, Poch O and Kieffer BL: Gene expression is altered in the lateral hypothalamus upon activation of the mu opioid receptor. Ann N Y Acad Sci 1129: 175-184, 2008

45. Yang YJ,LvSY,Xiu MH,XuN and ChenQ:Intracerebroventricular administration of apelin-13 inhibits distal colonic transit in mice. Peptides 31: 2241-2246, 2010.

46. Lutfy K, Eitan S, Bryant CD, Yang YC, Saliminejad N Walwyn W, Kieffer BL, Takeshima H, Carroll FI, Maidment NT and Evans CJ: Buprenorphine-induced antinociception is mediated by mu-opioid receptors and compromised by concomitant activation of opioid receptor-like receptors. J Neurosci 23 10331-10337, 2003.
47. Habata Y, Fujii R, Hosoya M, Fukusumi S, Kawamata Y, Hinuma S, Kitada C, Nishizawa N, Murosaki S, Kurokawa T, et al: Apelin, the natural ligand of the orphan receptor APJ, is abundantly secreted in the colostrum. Biochim Biophys Acta 1452: 25-35, 1999.

48. Masri B, Lahlou H, Mazarguil H, Knibiehler B and Audigier Y: Apelin (65-77) activates extracellular signal-regulated kinases via a PTX-sensitive G protein. Biochem Biophys Res Commun 290: 539-545, 2002.

49. Masri B, Morin N, Pedebernade L, Knibiehler B and Audigier Y: The apelin receptor is coupled to Gi1 or Gi2 protein and is differentially desensitized by apelin fragments. J Biol Chem 281: $18317-18326,2006$

50. O'Donnell LA, Agrawal A, Sabnekar P, Dichter MA, Lynch DR and Kolson DL: Apelin, an endogenous neuronal peptide, protects hippocampal neurons against excitotoxic injury. J Neurochem 102: 1905-1917, 2007.

51. Bai B, Tang J, Liu H, Chen J, Li Y and Song W: Apelin-13 induces ERK1/2 but not p38 MAPK activation through coupling of the human apelin receptor to the Gi2 pathway. Acta Biochim Biophys Sin (Shanghai) 40: 311-318, 2008.

52. Ji RR, Gereau RW IV, Malcangio M and Strichartz GR: MAP kinase and pain. Brain Res Rev 60: 135-148, 2009. 